\title{
Ultrasonographic diagnosis of fetuses with pentalogy of cantrell and turner syndrome, dual case reports and review of literature
}

\begin{abstract}
Background: Ultrasound imaging is an effective and routine diagnostic tool for identifying fetal anomalies. The congenital defect of Pentalogy of Cantrell, also known as Thoracoabdominal Ectopia, consists of 5 defects that include a cleft lower sternum, an anterior diaphragmatic defect, and the absence of the parietal pericardium, a connected or separate omphalocele, and a major cardiac anomaly. While rare, this congenital syndrome effects 5.5 of 1,000,000 live births, ultrasound imaging can be useful in identifying this defect in utero. Turner syndrome, or XO syndrome, affects 1 in 2500 to 1 in 3000 live born phenotypic female infants and is caused by a genetic abnormality of a single $\mathrm{X}$ chromosome. While characteristics of the syndrome can be observed in childhood, such as webbed neck, broad or pigeon chest, widely spaced nipples, multi-pigmented nevi, valgus of elbows, cardiac malformations and streak ovaries the syndrome often does not become apparent until functional deficits are noticed such as amenorrhea or short stature. While Turner syndrome requires genetic analysis for definitive diagnoses, similar to other congenital defects that are easily identified because of their gross morphological anomalies, ultrasound imaging offers an important tool for identifying XO syndrome. We present two such cases where US imaging was useful in making an early diagnosis of congenital anomalies.
\end{abstract}

Cases: Case 1 - pentalogy of Cantrell: A 33 year old woman, G2P1001, presented to clinic initially at 6 weeks and 5 days gestation for her first dating bedside ultrasound. The patient was treated for pregestational hypertension with methyldopa $500 \mathrm{mg}$ three times per day (TID) but she declined its use. She agreed to the use Labetalol $200 \mathrm{mg}$ TID as hypertensive therapy. A follow up first trimester ultrasound at 12 weeks and 6 days gestation showed the fetal heart beating outside to the thoracic cavity and the liver outside the abdominal cavity. The radiology impression was a ventral wall defect indicating likely Pentalogy of Cantrell. The patient was counseled about the very poor fetal prognosis and incompatibility with extrauterine life. She wished to proceed with pregnancy termination and underwent voluntary termination of pregnancy (VTOP) via dilation and curettage at 13 weeks and 6 days gestation.

Case 2 - turner syndrome: A 42 year old Caucasian female, Rh negative/antibody negative, G5P3013, at 15 weeks and 3 days gestation by last menstrual period (LMP) presented to our clinic for her first obstetric ultrasound. Bedside ultrasound showed a live intrauterine pregnancy and a corrected gestations age of 16 weeks 0 days and swelling around the abdominal wall and fetal head. The MFM ultrasound at 17 weeks 3 days gestation revealed fetal demise (FD), cystic hygroma and significant hydrops fetalis with bilateral pleural effusions and ascites. As the antibody screen was negative, the likely etiology was nonimmune hydrops most likely due Turner syndrome (45XO). The patient was induced with $400 \mathrm{mcg}$ of Misoprostol vaginally every 6 hours until delivery of a stillbirth female fetus.

Conclusion: Performing prenatal ultrasound imaging has become routine, beginning in the first trimester, with some conditions requiring more frequent assessments. US imaging is a valuable clinical tool, because it is non invasive has the potential to reveal critical clinical information and reassurance for the parents. This early detection offers both the clinicians and parent's options for managing the care of the developing fetus and the child after birth.

Keywords: Pentalogy of cantrell, Thoracoabdominal ectopia, Turner syndrome, XO syndrome
Volume 8 Issue 3 - 2017

\author{
Shadi Rezai,' Alexander C Hughes, ${ }^{2}$ Gregory \\ Hoge, ${ }^{2}$ Basem Z Bernaba,' Ray Mercado, ${ }^{4}$ \\ Paul N Fuller,' Cassandra E Henderson ${ }^{3}$ \\ 'Department of Obstetrics and Gynecology, Southern California \\ Kaiser Permanente, USA \\ ${ }^{2}$ St Georges University, School of Medicine, Grenada \\ ${ }^{3}$ Department of Obstetrics and Gynecology, Lincoln Medical \\ and Mental Health Center, USA \\ ${ }^{4}$ Department of Obstetrics and Gynecology, Comprehensive \\ Medical Care of the Bronx, USA
}

Correspondence: Cassandra E Henderson MD, Maternal Fetal Medicine, Department of Obstetrics and Gynecology, Lincoln Medical and Mental Health Center, 234 East 149th Street, Bronx, New York, 1045I, USA, Email rezsha@sgu.edu

Received: October 2I, 2017 | Published: December 2I, 2017

\section{Background}

Pentalogy of Cantrell or Thoracoabdominal Ectopia is a congenital syndrome characterized by a set of 5 congenital defects consisting of a
a. Mid-line supraumbilical abdominal wall
b. Lower sternum defect
c. Anterior diaphragm defect

\section{d. Diaphragmatic pericardium defect}

e. An intracardiac defect. ${ }^{1,2}$

The underlying pathophysiology of Pentalogy of Cantrell continues to be poorly understood but is thought to be related to defective formation and differentiation of the ventral mesoderm. ${ }^{3,4}$ Most importantly, mesoderm failure results in cardiac defects, which are the major determinant for severity and prognosis, most commonly 
ventral septal defect. ${ }^{4,5}$ Pentalogy of Cantrell is divided into two classes; class one is complete malformation with the presence of all five defects and classes two contains only four defects. ${ }^{4}$ Although this syndrome commonly results in still births, there have been reported cases of live births requiring immediate surgical repair. ${ }^{5}$ Yet, the survival rate is unknown as live cases are rare. ${ }^{5}$

Turner syndrome or XO syndrome is a genetic anomaly where the fetus only has a single $\mathrm{X}$ chromosome and occurs in 1 in 2500 to 1 in 3000 live born girls. ${ }^{6}$ If not diagnosed prenatally, then the syndrome may not be apparent during childhood and may only be considered during adolescence when the patient presents with a complaint of primary amenorrhea, lack of breast development or short stature. ${ }^{7,8}$ Other abnormalities include webbed neck, broad or pigeon chest, widely spaced nipples, multi-pigmented nevi, valgus deformity of the elbows, cardiac malformations and streak ovaries.

Both Turner syndrome and Pentalogy of Cantrell can be detected on routine prenatal ultrasound as early as 11 weeks. ${ }^{9,10}$ We present two cases of genetic abnormalities initially discovered on routine prenatal ultrasound.

\section{Presentation of cases}

\section{Case I - Pentalogy of cantrell}

A 33-year-old woman, G2P1001, presented to clinic initially at 6 weeks and 5 days gestation for her first dating bedside ultrasound. Previous obstetrical history includes one previous cesarean delivery, 4 years prior, for failure to progress. Past medical history was significant for pregestational/chronic hypertension and morbid obesity (body mass index of $50 \mathrm{~kg} / \mathrm{m} 2$ ). The first bedside ultrasound was used to correct gestational age and did not reveal any abnormalities. The patient was treated for pregestational hypertension with methyldopa $500 \mathrm{mg}$ three times per day (TID) but was non-compliant and was later switched to labetalol $200 \mathrm{mg}$ TID.

A subsequent follow up first trimester ultrasound at 12 weeks and 6 days gestation showed the fetal heart beating outside to the thoracic cavity and the liver outside to the abdominal cavity (Figure 1). A defined umbilical cord was seen. The radiology impression was a ventral wall defect, with the liver and heart observed outside the chest and abdominal cavity, indicating likely Pentalogy of Cantrell. The patient was counseled about the very poor fetal prognosis and incompatibility with extrauterine life. She wished to proceed with pregnancy termination and underwent voluntary termination of pregnancy (VTOP) via dilation and curettage at 13 weeks and 6 days gestation. The patient had an uncomplicated recovery.

\section{Case 2 - Turner syndrome}

A 42 year old Caucasian female, Rh negative/antibody negative, G5P3013, at 15 weeks and 3 days gestation by last menstrual period (LMP) presented to clinic for her first obstetric ultrasound. Bedside ultrasound showed a live intrauterine pregnancy and corrected gestational age of 16 weeks 0 days. Ultrasound also revealed presence of swelling around the abdominal wall and fetal head (Figure 2).

The patient was referred to the maternal fetal medicine service (MFM) for further evaluation of suspected fetal fluid collection as noted above. The MFM ultrasound at 17 weeks 3 days gestation revealed intrauterine fetal demise (IUFD), massive cystic hygroma and significant hydrops fetalis with bilateral pleural effusions and ascites consistent with hydrops fetalis. The ultrasound suggested a female fetus. As the antibody screen was negative, suggesting nonimmune hydrops, most likely due to fetal Turner syndrome (45XO). Patient declined noninvasive prenatal testing (NIPT) or amniocentesis to confirm diagnosis. She also declined dilatation/evacuation for termination and opted for induction of labor due to fetal demise. The patient was induced with $400 \mathrm{mcg}$ of Misoprostol vaginally every 6 hours until delivery of a stillbirth female fetus. The remainder of her hospital course was uncomplicated.

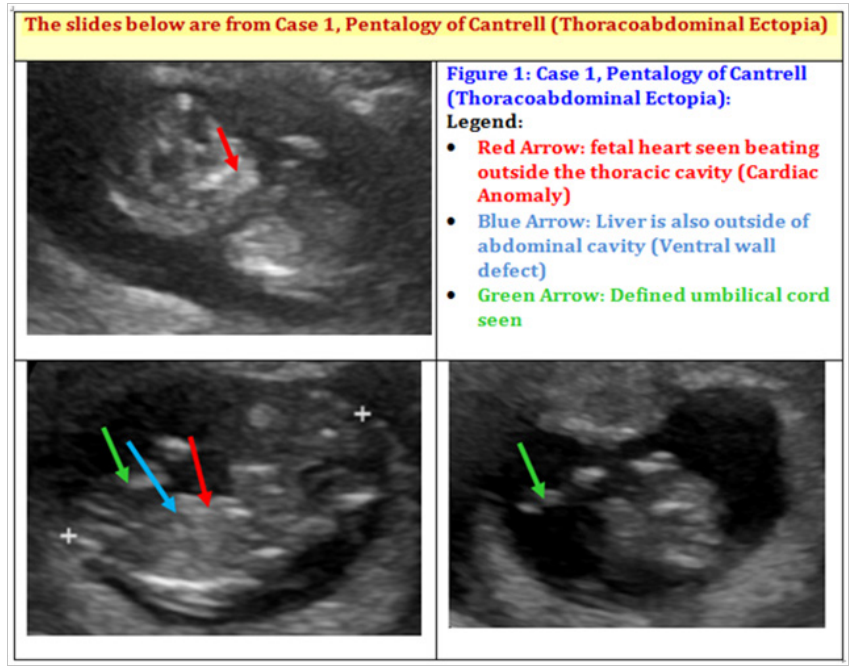

Figure I Case I, Pentalogy of Cantrell (Thoracoabdominal Ectopia): First trimester ultrasound at 12 6/7 weeks showed fetal heart seen beating outside the thoracic cavity. Liver is also outside of abdominal cavity. Defined umbilical cord seen. Impression:Ventral wall defect - likely Pentalogy of Cantrell.

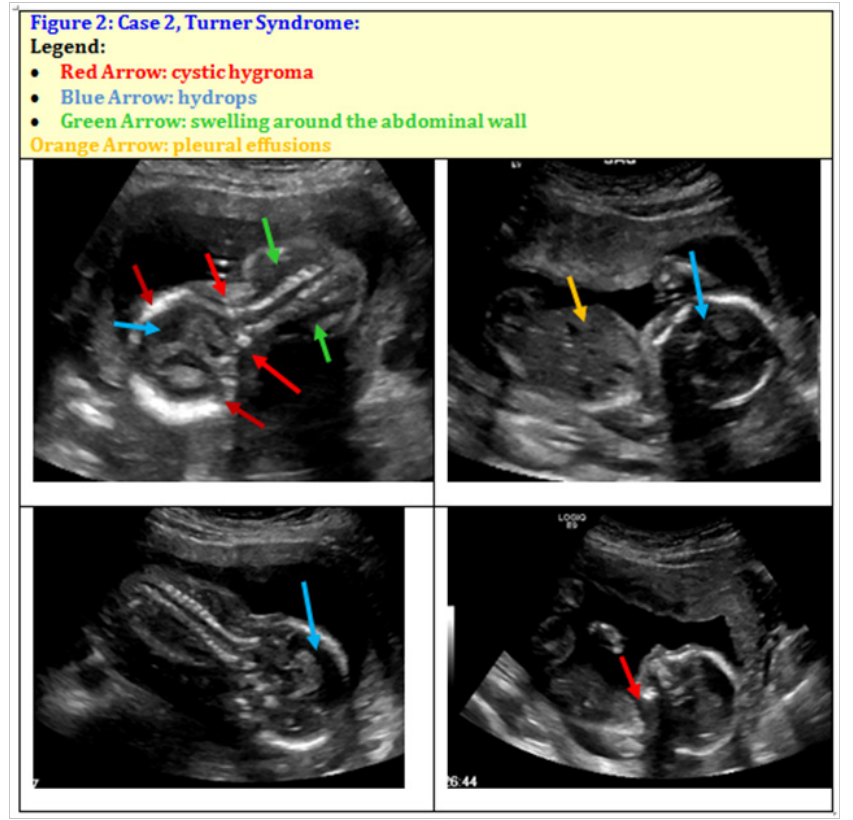

Figure 2 Case 2, Turner Syndrome: Ultrasound revealed intrauterine fetal demise (IUFD), Massive cystic hygroma, significant hydrops with bilateral pleural effusions and ascites seen as presence of swelling around the abdominal wall and swelling around fetal head, Probable female fetus.

\section{Discussion}

Routine ultrasound can detect many morphological anomalies including both structural and genetic defects. Pentalogy of Cantrell can be detected by ultrasound as early as 10 weeks. ${ }^{4}$ Common findings are omphalocele and ectopic cordis but must also include pericardial 
defect and an intra cardiac defect for the diagnosis. ${ }^{2}$ Ectopic cordis is suspected when cardiac contractile activity is detected outside the chest cavity and can be confirmed by Doppler. ${ }^{10,11}$ The thoracoabdominal type of ectopic cordis is specific to Pentalogy of Cantrell and is highly suggestive for diagnosis. ${ }^{5}$ The thoraco-abdominal type of ectopic cordis is defined by a ventral wall defect which extends to the umbilicus causing the diaphragm to have a ' $\mathrm{V}$ ' shaped defect. ${ }^{12}$ The omphalocele can be visualized when multiple bowel loops are seen outside the abdominal cavity, with the cavity itself measuring smaller than expected for gestational age. ${ }^{11}$

Although two dimensional ultrasound is adequate for diagnosis of Pentalogy of Cantrell for a skilled clinician some have advocated for the use of 3D ultrasound or MRI which allows for more detailed visualization. ${ }^{13}$ In the case described above conventional ultrasound was adequate for a diagnosis of Pentalogy of Cantrell by visualization of extra-thoracic fetal heart beats (Figure 1 red arrow), extraabdominal liver, and a ventral wall defect (Figure 1 blue arrow).

Similarly, Turner syndrome can be detected on ultrasound but initial findings are less specific than with Pentalogy of Cantrell. Ultrasound findings associated with Turner syndrome are cystic hygroma and fetal hydrops in a female fetus..$^{9,14,15}$ A cystic hygroma is an enlargement of lymphatic ducts and accumulation of lymph causing increased nuchal translucency. ${ }^{9,16}$ Fetal hydrops is defined as greater than 2 of the following; ascites, pleural effusions, pericardial effusion, or generalized and skin edema. ${ }^{16}$ Nuchal thickness is a common initial finding, as in this case, and may be accompanied by hydrops fetalis leading to fetal demise. Findings in this case included a massive cystic hygroma (Figure 2 red arrow), significant hydrops (Figure 2 blue arrow) with bilateral pleural effusions (Figure 2 orange arrow) and ascites. Although diagnosis was not confirmed with postmortem genetic testing (per patient request) these findings, along with spontaneous fetal demise, are highly suggestive of Turner syndrome. ${ }^{14}$

Unlike Pentalogy of Cantrell, which is a series of malformations, Turner syndrome is an inherent genetic anomaly which cannot be repaired. Because Turner syndrome is of a genetic origin, definitive diagnosis must be made through fetal tissue karyotype testing, and cannot be made solely with ultrasound, however pentalogy of Cantrell may be diagnosed by visualization. ${ }^{15}$ Once born, Turner syndrome individuals face a host of abnormalities including metabolic, cardiac and reproductive. Compared to pentalogy of Cantrell which requires immediate surgical repair and rarely live beyond the age of 10 , Turner syndrome patients can live into adulthood. ${ }^{5,8,17}$

\section{Conclusion}

Ultrasound has become a routine component of prenatal care. With the frequency of exams being dictated by specific indications. Ultrasound is non-invasive, allows the clinician to attain some basic important information about the pregnancy and can be reassuring to parents. Because ultrasound can reveal morphological anomalies, it remains a useful screen for congenital malformations of all causes. Thus, it can be used for detecting defects such as the Pentalogy of Cantrell, but additionally, can also be useful in detecting genetic abnormalities such as Turner Syndrome, which often may not present until much later in life during adolescence.

Early identification of many obstetric or conditions prenatally can be beneficial. Both for clinicians to plan early intervention or future follow up and for parents to consider their options including elective termination or expectations about viability of pregnancy.

\section{Acknowledgements}

The authors would like to thank Ms. Judith Wilkinson, Medical Librarian at Lincoln Medical and Mental Health Center Science Library for providing the reference articles.

\section{Conflicts of interest}

The Authors did not report any potential conflicts of interest.

\section{References}

1. Ramasauskaite D, Snieckuviene V, Zitkute V, et al. A Rare Case Report of Thoracic Ectopia Cordis: An Obstetrician's Point of View in Multidisciplinary Approach. Case Rep Pediatr. 2016;2016:5097059.

2. Sowande OA, Anyanwu LJ, Talabi AO, et al. Pentalogy of Cantrell: a report of three cases. J Surg Tech Case Rep. 2010;2(1):20-23.

3. Çelik Y, Hallioğlu O, Basut N, et al. A rare case of cardiac anomaly: prenatally diagnosed ectopia cordis. Turk Pediatri Ars. 2015;50(2):129131.

4. Naburi H, Assenga E, Patel S, et al. Class II pentalogy of Cantrell. BMC Res Notes. 2015;8:318.

5. Pius S, Abubakar Ibrahim H, Bello M, et al. Complete Ectopia Cordis: A Case Report and Literature Review. Case Rep Pediatr. 2017;2017:1858621

6. Sybert VP, McCauley E. Turner's syndrome. $N$ Engl J Med. 2004;351(12):1227-1238

7. Koeberl DD, McGillivray B, Sybert VP. Prenatal diagnosis of 45,X/46,XX mosaicism and 45,X: implications for postnatal outcome. Am J Hum Genet. 1995;57(3):661-666.

8. Morgan T. Turner syndrome: diagnosis and management. Am Fam Physician. 2007;76(3):405-410.

9. Surerus E, Huggon IC, Allan LD. Turner's syndrome in fetal life. Ultrasound Obstet Gynecol. 2003;22(3):264-267.

10. Türkçapar AF, Sargın Oruc A, Öksüzoglu A, et al. Diagnosis of pentalogy of cantrell in the first trimester using transvaginal sonography and color Doppler. Case Rep Obstet Gynecol. 2015;2015:179298.

11. Naren Satya SM, Mayilvaganan KR, Prathyusha IS, et al. A Recurrent Case of Pentalogy of Cantrell: A Rare Case with Sonological Findings and Review of Literature. Pol J Radiol. 2017;82:28-31.

12. Kumar B, Sharma C, Sinha DD, et al. Ectopia cordis associated with Cantrell's pentalogy, Ann Thorac Med. 20083(4):152-153.

13. Yew MH, Fishman EK, Vogel-Claussen J, et al. Assessment of Pentalogy of Cantrell using 3D Multidetector Computed Tomography. Radiol Case Rep. 2016;4(1):273.

14. Goulart VV, Liao AW, Carvalho MH, et al. Intrauterine death in singleton pregnancies with trisomy 21, 18, 13 and monosomy X. Rev Assoc Med Bras (1992). 2016;62(2):162-170.

15. Munteanu O, Cîrstoiu MM, Filipoiu FM, et al. Morphological and ultrasonographic study of fetuses with cervical hygroma. A cases series. Rom J Morphol Embryol. 2016;57(4):1421-1427.

16. Norton ME, Chauhan SP, Dashe JS. Society for maternal-fetal medicine (SMFM) clinical guideline \#7: nonimmune hydrops fetalis. Am J Obstet Gynecol. 2015;212(2):127-139.

17. Practice Bulletin No. 162: Prenatal Diagnostic Testing for Genetic Disorders. Obstet Gynecol. 2016;127(5):e108-e122. 